\title{
Dans le halo des MOOC, la rationalité communicationnelle de la formation 2.0
}

In the halo of MOOC, the communicational rationality of the 2.0 training

\section{Carsten Wilhelm}

\section{(2) OpenEdition}

\section{Journals}

Édition électronique

URL : http://journals.openedition.org/communicationorganisation/5215

DOI : 10.4000/communicationorganisation. 5215

ISSN : $1775-3546$

Éditeur

Presses universitaires de Bordeaux

\section{Édition imprimée}

Date de publication : 1 juin 2016

ISBN : 979-10-300-0059-7

ISSN : 1168-5549

\section{Référence électronique}

Carsten Wilhelm, «Dans le halo des MOOC, la rationalité communicationnelle de la formation 2.0 » Communication et organisation [En ligne], 49 | 2016, mis en ligne le 01 juin 2019, consulté le 02 janvier 2020. URL : http://journals.openedition.org/communicationorganisation/5215 ; DOI : 10.4000/ communicationorganisation.5215 


\title{
Dans le halo des MOOC, la rationalité communicationnelle de la formation 2.0
}

\author{
Corsten Wilhelm ${ }^{1}$
}

Notre système scolaire sera complètement bouleversé dans les dix prochaines années.

Thomas Edison (1913) à propos de l'invention du cinéma²

Le progrès dans le domaine des TIC va faire disparaître l'université résidentielle d'ici 2030; elle va être remplacée par des technologies d'apprentissage personnalisées.

Peter F. Drucker (1997) ${ }^{3}$.

\section{Les règles du jeu de la «transformation digitale»}

Régulièrement annoncées comme une révolution transformant les secteurs économiques et sociétaux le plus variés, les technologies numériques, ces "game changers»", sont également positionnées et promus (Jaillet 2014) comme agents de rupture dans les discours d'accompagnement liés à leur application dans le secteur de la formation, à l'université comme en entreprises, dans le cadre de la "transformation digitale ${ }^{5}$ à laquelle les organisations consacrent désormais un quart de leur budget informatique ${ }^{6}$. D'après le baromètre de "l'e-learning en France» de l'association française des industriels du numérique de l'éducation et de la formation (AFINEF

\footnotetext{
1 Carsten Wilhelm, Cresat (EA 3436), Université de Haute Alsace. Domaines de recherches : l'impact du numérique sur les usages sociaux des médias, la formation à distance, ainsi que l'approche interculturelle dans les sciences de l'information et de la communication ; carsten.wilhelm@uha.fr

2 Edison cité dans Saettler $(1968,68)$ (original en anglais, notre traduction).

3 Drucker, Forbes Magazine 1997 (original en anglais, notre traduction).

4 L'expression anglais "Game changer" - modificateur du jeu- est très révélatrice à cet égard. Diane Oblinger, présidente d'Educause, organisme non commercial pour la promotion de l'utilisation des technologies dans l'éducation, a, par exemple, repris cette expression pour le titre d'une publication de 2012 sur l'impact du numérique dans l'apprentissage.

$5 \mathrm{Ce}$ terme, très présent dans le discours des consultants n'a été que très peu sujet de traitements scientifiques, particulièrement en SIC. L'entreprise Futureskill, par exemple, l'applique spécifiquement à la formation d'entreprise.

6 Baromètre des investissements numériques, Accenture, novembre 2015. Il faut évidemment citer des données issues d'études commanditées par les acteurs économiques du champ en question avec prudence.
} 
$2015)^{7}, 90 \%$ des entreprises françaises utiliseraient l'e-learning, pour des raisons essentiellement économiques (réduction de coût, échelle et vitesse de formation, budget constant).

Forme particulière des dispositifs numériques de formation, les MOOC jouissent depuis 2012 d'une visibilité importante dans les médias, essentiellement générée par la médiatisation initiale de quelques exemples prestigieux dont le fonctionnement s'est avéré proche des productions de l'industrie culturelle (Wilhelm 2015). Aujourd'hui le paysage s'est davantage diversifié et si l'universalité des MOOC - au sens démocratisation et adaptation à tout public - semble mise en cause par l'aveu de la faiblesse de ses promoteurs mêmes ${ }^{8}$, des formes secondaires, dites plus adaptées au fonctionnement des entreprises, sont promues actuellement. Une fois le terrain préparé par les MOOC grand public, il s'agit maintenant pour les acteurs positionnés de déployer leur savoir faire et d'implémenter cette forme avec toute sa symbolique au service de l'entreprise, discours d'accompagnement aidant. L'idéologie initiale se transforme alors en business model.

Les MOOC ont donné lieu à des analyses et aides mises en perspectives critiques dans des disciplines académiques variées (Karsenti 2013; Moeglin 2014; Collet et Wilhelm 2015). La nature dispositive de nos objets d'étude, qui s'attache à comprendre la communication dans la complexité en relation aux différentes dimensions du dispositif (Jacquinot et Joplin 2002; Wilhelm 2010), permet d'en faire des «instruments de la rationalité en finalité " tout autant qu'un "espace d'émancipation.» (Berten 1999: 45). Nous proposons ainsi de les explorer ici à travers le prisme des actions rationnelles en valeur et en finalité de Max Weber (2004) ${ }^{9}$, comprises respectivement comme normatives, au sens «agir pour la valeur intrinsèque d'une action» et performatives, au sens performance, «agir pour obtenir un résultat», qu'il soit financier, de pouvoir ou d'autre nature, après un choix rationnel parmi des alternatives à disposition.

Notre texte procède en deux temps. Dans un premier temps, nous revenons sur l'histoire de l'enseignement à distance et en ligne afin d'apporter une dimension diachronique à des phénomènes qui dans leur posture de rupture semblent exister sui generis à chaque nouvelle apparition et qui, chacun à sa manière, porte en lui ce double caractère de rationalité.

Dans un deuxième temps, nous souhaitons, appuyés sur notre analyse «médiaculturelle» des MOOC (Wilhelm, 2015), souligner les liens avec leurs dérivés récents, les $\mathrm{COOC}$, SOOC, et autre SPOC, et ainsi dessiner les enjeux en évolution. Par ce traitement nous proposons alors de situer la

7 Association des professionnels dédiée à la promotion du numérique dans léducation.

8 https://pando.com/2014/05/12/a-qa-with-godfather-of-moocs-sebastian-thrun-after-he-disavowed-his-godchild/ (consulté 10/10/15) mais aussi https://dzone.com/articles/has-the-mooc-experiment-failed (consultés 22/11/15)

9 Et en particulier suivant son application par Olivier Galibert (2014) au lien communautaire en ligne. 
médiation numérique du savoir dans les organisations comme un défi pour les SIC, néanmoins bien armées pour une analyse critique de ce phénomène, aidées particulièrement par leur ouverture aux sciences de l'éducation et à la sociologie des médias.

Nous retiendrons comme point de départ de notre réflexion un travail de recherche sur la formation en ligne interculturelle ${ }^{10}$. Le suivi régulier du champ en question a ensuite débouché sur l'analyse d'un corpus sélectionné de textes sur l'univers des MOOC publiés aussi bien dans la sphère académique ainsi que dans la presse spécialisée en ligne. Récemment nous avons pu le compléter par plusieurs entretiens semi-directifs avec des acteurs de ce secteur (tuteurs, community managers, ingénieurs pédagogiques, coordinateurs de formation). Nous avons pu également observer de l'intérieur le fonctionnement de plusieurs MOOC comme apprenant (français, allemands, anglais et américains) et comme expert-contenu (MOOC DCW). Dans la suite de ce travail nous souhaitons explorer les dispositifs en question en profondeur sur la base exposée ici, par des études de cas approfondis et en analysant les fonctions métiers en jeu.

\section{Retour sur l'ambivalence de l'histoire de la formation à distance et en ligne}

There must be an 'industrial revolution' in education, in which educational science and the ingenuity of educational technology combine to modernize the grossly inefficient and clumsy procedures of conventional education. Work in the schools of the future will be marvelously though simply organized, so as to adjust almost automatically to individual differences and the characteristics of the learning process. There will be many laborsaving schemes and devices, and even machines - not at all for the mechanizing of education, but for the freeing of teacher and pupil from educational drudgery and incompetence. Sidney Pressey 1933, p. 582-583

Cette citation de Sidney Pressey, professeur de psychologie et inventeur d'une " machine à enseigner » ${ }^{11}$, illustre parfaitement la collusion des objectifs normatifs et performatifs de progrès des technologies dites éducatives, action rationnelle qui vise à la fois la performance des institutions et en même temps la libération des acteurs individuels de leurs propres défauts. À la lumière de cette citation, il paraît opportun de retracer de manière synthétique le développement de l'enseignement à distance (EAD) sous deux aspects. Si nous admettons le primat de l'histoire institutionnelle et de ses discours (Thibault 2013) - et non de la technologie seule - sur l'évolution de la EAD, il est néanmoins incontournable, dans une perspective médiaculturelle (Maigret et Macé 2005), d'aborder la question sous l'angle de la place des technologies

$10 \mathrm{C}$. Wilhelm, Processus interculturels et apprentissage à distance : de la communication à la communauté, Thèse, Dijon : Université de Bourgogne, 2009.

11 Hilgard E. R. \& Bower G. H. 1966. Theories of learning. 3rd ed, New York:Appleton-Century-Crofts. Chapter 16: Learning \& the technology of instruction, 554-561 Programmed learning. 
et médias utilisés, de l'usage des TIC pour la formation et de leur combinaison récente afin de pouvoir prendre en compte les aspects communicationnels au prisme de leur double action rationnelle. Nous n'avançons pas un argumentaire qui lie l'apparition de technologies au progrès dans une visée eschatologique des formes sociétales. Nous insistons, tout au contraire, sur l'inscription des technologies dans des projets plus larges dont ils sont des outils. On pointera dans notre exposé au moins trois motivations pour introduire des innovations technologiques dans l'enseignement : la motivation économique : l'accès à un nouveau marché, la motivation sociale et étatique: améliorer l'instruction des citoyens (social management) ainsi que la motivation par nécessité et opportunité suite à la massification de l'enseignement supérieur et à des ressources en diminution ${ }^{12}$. Ce sont ces facteurs de motivation, liés aux opportunités technologiques et aux idéologies de rupture et d'opposition aux institutions qui les portent (Flichy 2001, Thibault 2002), manifestes dans les discours et les pratiques, qui permettent d'approcher le phénomène de l'enseignement en ligne, et des MOOC en particulier, par la rationalité communicationnelle.

\section{L'enseignement par correspondance}

Le 20 mars 1728, la Boston Gazette contient la publicité suivante: Caleb Phillips, professeur de la nouvelle méthode de sténographie, fait savoir que toute personne vivant à la campagne désireuse d'apprendre cet art peut, en se faisant envoyer plusieurs leçons par semaine, être aussi bien instruite que ceux qui habitent Boston ${ }^{13}$. Il est intéressant de relever ici l'usage du mot «instruite» qui indique le modèle pédagogique ${ }^{14}$-l'étudiant, qui est " désireux d'apprendre un art », est essentiellement passif, il est instruit-ainsi que l'argument commercial qui brandit une nouvelle méthode et qui s'adresse aux personnes vivant à la campagne, loin des nouveaux centres intellectuels et économiques ${ }^{15}$, grâce à un procédé de communication relativement nouveau. Un siècle plus tard, en 1840, Issac Pitman propose ses cours de comptabilité et de sténographie par correspondance aux Anglais. L'Europe continentale voit naître le premier institut de formation à distance à Berlin en 1856, créé par Charles Toussaint et Gustav Langescheidt, pour l'apprentissage des langues. En France, c'est à partir de 1877 que sont créés les «cours Hattemer», «L'école chez soi»(Eyrolles 1891), «École universelle» (1907). Le public visé est d'abord

12 David Noble (2001) nous rappelle les risques liés à la tentation du faible coût et de la massification qui resurgit de nouveau avec les nouvelles modalités de formation de nos dernières décennies. Il s'intéresse en premier lieu aux développements en Amérique du Nord au début du siècle dernier.

13 Battenberg (1971) dans Holmberg (1995), 48.

14 Le terme est présent encore aujourd'hui - par exemple dans le champ du instructional design qui est très proche de l'ingénierie pédagogique sans pour autant vouloir dire la même chose.

15 Une problématique qui revient régulièrement et qui est encore d'actualité aujourd'hui: l'absence de réseaux rapides en milieu rural donne régulièrement lieu à des initiatives alternatives pour acheminer le savoir (satellite, WIMAX ...). 
un public relativement aisé qui éprouve le besoin d'une formation spécialisée hors cadre scolaire. Toutes ces initiatives viennent d'organismes privés dont les objectifs sont l'enseignement de la culture générale, des arts, des langues étrangères, le soutien scolaire et la préparation aux concours administratifs. La logique initiale était de trouver un modèle économique en proposant l'accès au savoir, à la culture ou d'offrir une compétence pratique à une clientèle aisée mais empêchée, susceptible de tirer profit des modalités désincarnées de l'apprentissage en raison de son éducation et de sa socialisation. Il existe pourtant des exemples d'autres motivations pour d'autres publics : ainsi en 1873, Anna Eliot Ticknor crée aux États-Unis la Société d'encouragement des études à la maison, la " Society to Encourage Study at Home ", destinée principalement à la clientèle féminine. Les étudiants étaient majoritairement des femmes. L'accès des femmes à la formation et à l'enseignement était d'ailleurs pour Ticknor un des objectifs importants de son action. Le secteur public, avec sa mission de garantir l'accès à l'éducation supérieure à un coût abordable, ne tardera pas à s'intéresser ensuite à l'enseignement à distance. En 1858, L'université de Londres développe son système d'études « externes » en collaboration avec d'autres collèges qui profitent de ses cours par correspondance. En 1874, l'Illinois Wesleyan University met en place un programme permettant l'obtention de bachelors (équivalent de l'ancien DEUG ou DU dans le système universitaire français) et de "graduate degrees» (Licence, Master d'aujourd'hui) in absentia.

$\mathrm{Si}$, d'un point de vue microsociologique, ces offres relèvent d'abord du marché et puis des universités, les raisons de l'émergence de ces initiatives renvoient à des changements sociaux et à des changements de valeurs macrosociologiques. Nous pouvons alors apercevoir la tension entre action rationnelle en valeur, l'appui à l'individu dans une société changeante, et l'action rationnelle en finalité, liée à l'activité économique des deux côtés de ce marché. Il devenait important de donner des moyens de formation à des groupes à croissance exponentielle qui migraient vers les métropoles dans un élan de management social. En même temps, les orientations libérales mettaient l'individu au centre des préoccupations et misaient sur sa capacité à s'élever grâce à l'éducation. Cette attitude émancipatoire est plus que jamais d'actualité aujourd'hui quand l'accent est mis sur l'individualisation, dont rêvait Pressey, aux dépens des institutions, réduisant ainsi le positionnement de l'éducation comme bien public. Les entreprises ont donc été au premier rang des acteurs de la formation à distance. Ceci semble changer quelque peu avec l'usage des médias multiples du siècle dernier, adopté en particulier par le secteur public ${ }^{16}$.

16 C'est seulement à partir du XX $X^{\mathrm{e}}$ siècle que la création, dans plusieurs pays d'Europe, de ministères du Travail ainsi que l'intérêt éprouvé des gouvernements nationaux pour l' "instruction nationale pour tous », font entrer la modalité « par correspondance » dans la panoplie des moyens de formation des citoyens. 


\section{L'utilisation croissante des médias multiples}

Alors que l'introduction de la radio dans les dispositifs d'enseignement à distance apparaît d'abord comme un échec relatif (Jeffries 2000), l'introduction de la télévision semble correspondre à un vrai besoin. Dès 1932, l'université de l'État d'Iowa transmet des émissions et obtient en 1950 une licence de diffusion pour émettre la première chaîne de télévision éducative non expérimentale (Chaptal 2003).

Cette introduction de la télévision est le point crucial à partir duquel se met en place une alternative à l'enseignement par correspondance traditionnelle : l'usage des contenus audiovisuels et multimédia. La première université publique à proposer l'enseignement à distance diplômant est, dès 1962, l'UNISA (University of South Africa). Institutionnellement, cette phase est marquée par la création d'universités ouvertes, d'abord en GrandeBretagne à partir de 1969 : l'Open University (OU), installée en Angleterre à Milton Keynes - sorte de ville nouvelle -, a été le précurseur et l'exemple emblématique d'une série de créations du même type, qui dans les années soixante marquaient l'envie de faire profiter un maximum de personnes sans qualification d'un système ouvert, et menant aux plus hauts diplômes. Dès lors, les universités d'État, à l'instar des grandes institutions de statut privé, vont proposer des parcours diplômants. Selon Sir John Daniel $(1997,2012)$, vice-chancelier de l'OU de 1990 à 2001, ces créations d'universités ouvertes sont la plus grande réussite du télé-enseignement avec, pour les douze plus grandes, plusieurs millions d'inscrits et pour la seule Open University 200.000 étudiants dès sa création, soit plus que dans toutes les universités britanniques réunies. Selon Daniel, les trois défis actuels des systèmes éducatifs, favoriser l'accès à l'éducation, augmenter la qualité de l'éducation et baisser le coût de l'éducation, ont des conséquences importantes pour le développement des nouvelles formes médiatiques dans l'enseignement. Selon lui, ce sont les technologies au service de l'éducation qui peuvent relever ces défis ${ }^{17}$. Ces mêmes arguments servent de discours d'accompagnement et de légitimation, transformant la vague des MOOC, forts utilisateurs de l'audiovisuel, dans une action rationnelle en valeur pour ses parties prenantes potentielles.

\section{L'avènement de la FOAD et des technologies interactives}

L'apparition de lordinateur personnel, puis de la télématique, puis des hypermédias et du web modifie encore une fois la donne et représente un saut paradigmatique (Power 2007; Koschmann 1996), à travers la mise en valeur de l'interaction pédagogique dans les deux sens, associée à des modalités ouvertes d'apprentissage. Lenseignement à distance " interactif " et " collaboratif ", couplé à l'externalisation cognitive croissante de certains aspects du traitement de l'information et de la connaissance engendre une démocratisation de

17 http://www.educationandcareernews.com/higher-education/sir-john-daniel-today-access-to-higher-educationis-potentially-universal 
la production et de l'accès au savoir et une désintermédiation, c'est-à-dire la disparition d'intermédiaires entre connaissances et apprenants. Cette orientation est au cour de ce que l'on a désormais tendance à appeler des cMOOC, ou MOOC collaboratifs, qui s'appuient essentiellement sur la mise en relation des apprenants au lieu d'une transmission de contenu de haut en bas. Le slogan «l'apprenant au centre » devient alors lui-même central et donne à la fois la justification et le programme des développements et dispositifs à imaginer. Mais y a-t-il ici une véritable différence avec les débuts de l'EAD ? Son but initial nétait-il pas déjà le même ? Les opportunités offertes par les innovations technologiques sont difficilement exploitables en vue de la réalisation de la promesse d'ouverture et d'émancipation de l'individu ${ }^{18}$.

Que retenons-nous à ce stade? Tout d'abord,les deux éléments caractéristiques des débuts de l'EAD et dont on parle aujourd'hui encore, sont la médiation d'un contenu et la médiation d'une relation enseignant-enseigné (Holmberg 1983). La modalité pédagogique généralement employée est soit l'autoformation simple, par laquelle l'apprenant consulte les documents envoyés, soit une relation bilatérale directe apprenant/professeur- correcteur, quand le cours contient des devoirs à renvoyer. La plupart du temps, le modèle pédagogique est transmissif et utilise le canal du courrier pour la simple transmission de contenu et d'exercices mais c'est bien comme l'affirme Wallet $(2006,93)$ la « facilité de l'accès à la connaissance [qui] favorise les utopies pédagogiques et entretient tout un discours eschatologique ".

Dans cette optique, en effet, se pose la question de savoir si la conception de la classe à distance (classe virtuelle, webinars) ou plus spécifiquement les MOOC ou SPOC ne représentent pas, eux aussi, cette caractéristique intraparadigmatique, mus par un discours les situant étroitement dans la sphère d'action rationnelle en valeur, tout en servant une action rationnelle en finalité basée sur des modalités existantes.

Ainsi, les questions suivantes se posent:

Comment situer d'un point de vue communicationnel les phénomènes très récents évoqués ? Comment conceptualiser leurs caractéristiques? Quelle grille de lecture des innovations numériques dans les technologies de formation? Quelle approche critique pour faire face à la collusion de valeurs et intérêts qui dépassent les discours d'accompagnement et infusent les pratiques? La grille d'analyse des médiacultures (Maigret et Macé 2005) nous permet de discerner les éléments fonctionnels des nouveaux dispositifs, qui finalement ressemblent beaucoup les anciens. Elle appelle alors à une méthodologie ellemême dispositive (Wilhelm 2010).

18 Sans mentionner que l'instrumentalisation de l'interaction en communauté entre apprenants n'entraine pas automatiquement une situation d'apprentissage améliorée (Galibert 2014,129). 


\section{Comprendre les évolutions des dispositifs numériques de formation}

Une grille de lecture dispositive: vers une médiaculture des formations numériques

Les médiacultures (en tant qu'objet) sont comprises comme des « ressources culturelles importantes dans les processus d'acculturation qui sont typiques des sociétés transnationales de la seconde modernité " (Macé 2005). Quant au MOOC, il s'agit de terrains qu'on pourrait assimiler aux produits de l'industrie culturelle, partageant avec elle des outils (production audiovisuelle, jeux vidéos), ses acteurs (sociétés de production, auteurs, scénaristes) et des canaux de diffusion (web, applications mobiles). Concrètement, dans le cas d'un dispositif de type MOOC, il s'agit de l'utilisation des technologies numériques dans les processus d'apprentissage en ligne selon une modalité spécifique et, du moins dans le scénario officiellement admis, selon ces critères minimaux : des contenus numériques (audiovisuels, souvent, textuels, parfois, interactifs plus rarement) sont organisés en ligne pour faire " cours ", un accès gratuit est organisé par simple inscription (création de profil, suivi des données de connexion), cet accès numérique individuel permettant une participation en tout lieu et hors institution.

Ce phénomène est également emblématique d'un accès démocratique à une forme de la culture numérique et à la circulation du savoir. L'acronyme MOOC en lui-même est d'ailleurs éloquent, combinant une vision alternative, ouverte (open) avec une modalité existante (online courses) tout en promettant en effet d'échelle d'augmentation (massif), présent dans d'autres secteurs également ( i ipad rollout », déploiement de TBI etc.). Ce système de valeurs des MOOC fait suite aux utopies info-documentaires de Paul Otlet et Vannevar Bush et à l'idéologie californienne (Turner 2012) valorisant l'idée d'un accès à la connaissance et à l'apprentissage individuel choisi, dans une ambiance de mépris des institutions établies, jugées inadaptées, hégémoniques et rigides ${ }^{19}$.

La démocratisation de l'accès au savoir par les cours ouverts en ligne rappelle la démocratisation de la culture par les industries culturelles ainsi que "l'utopie jacobine d'une 'populiculture' par la télévision monopoliste d'état et l'exaltation républicaine du passage d'une classe de 40 élèves à une classe de 40 millions d'élèves » (Macé 2005). Cette vision d'une démocratisation par l'accès prend racine dans un espace public pourtant fragmenté (Miège 1995, 1997), caractérisé par l'industrialisation et la marchandisation de la culture et de la connaissance. Le discours en faveur des MOOC mobilise cet argument tout en y ajoutant l'aspect individualisant du numérique et sa capacité de mise en réseau, souvent sous le terme "rupture ».

19 L'application de cette idéologie au secteur éducatif trouve une partie de son inspiration dans la pédagogie critique de Paolo Freire, Ivan Illich, Michael Apple et Henry Giroux (Downes 2012), http://halfanhour. blogspot.fr/2012/04/rise-of-moocs.html 
La culture numérique de formation en entreprise : MOOC, SOOC, COOC et SPOC, sur quelle étagère?

Les entreprises sont aujourd'hui intéressées à profiter de ces injonctions variées de plusieurs manières distinctes:

Loffre des MOOC grand public ou $\mathrm{SOOC}^{20}$ existants - universitaires pour la plupart - peut être un choix pour les cadres pour une formation individuelle et une progression de carrière, mais ce choix a des limites importantes. Comment évaluer le degré d'adaptation aux besoins localement constatés? Quelle valorisation concrète dans les carrières - pour les apprenants et pour les enseignants?

L'entreprise peut créer un "MOOC d'entreprise » (COOC pour Corporate open online cours) afin de communiquer son " esprit d'innovation » et de diffuser sa marque à l'extérieur tout en cultivant une " culture d'entreprise " par un storytelling légitimé par sa valeur de formation. Ici, on insiste bien sur l'aspect reconnaissance auprès des prospects (clients, partenaires, employées), proche des salariés ambassadeurs sur les réseaux sociaux (Larroche 2013). Il s'agit surtout d'une répétition des structures et des objectifs principaux: marketing - visibilité de la marque et de la culture de l'entreprise ${ }^{21}$ - et recrutement - sourcing et assessment (Karsenti 2013) ${ }^{22}$. Leffet " halo ", produit par l'injonction à « l'ouverture », « l'innovation », voire la " rupture », participe à l'attrait de cette possibilité, qui est, par son inscription symbolique, une action rationnelle en finalité déguisée en valeur.

L'entreprise peut également souscrire à des formations "sur mesure », inspirées des MOOC, mais adaptées au cadre de la formation professionnelle dont l'individualisation est un des aspects les plus importants. Les SPOC (Small private open online courses), en particulier, sont censés remplir ce rôle. Les acteurs du secteur sont alors en droit de considérer que cette dernière option manque de profondeur et d'innovation car, sans le bonus d'ouverture ni la qualité «massive », si caractéristique des MOOC, ce type de formation revient à l'e-learning classique, l'usage du numérique et de ressources en ligne dans des dispositifs variables, pour la formation des salariés ${ }^{23}$ pour laquelle la production sur « étagère » de ressources disponibles à la demande devance les MOOC/SPOC et où l'usage de cours « sur mesure » est aujourd'hui majoritaire ${ }^{24}$.

20 Specialized Open Online Course - cours en ligne toujours ouverts mais très spécialisés et par défaut à public réduit.

21 Citons comme exemple Orange, BNP Parisbas, en France ou encore SAP en Allemagne.

22 http://www.latribune.fr/blogs/mooc-and-co/20140409trib000824439/pourquoi-les-entreprises-se-lancentdans-les-mooc-.html

23 Nous ne mettons par ailleurs nullement en cause le potentiel de ce type de formation (e-learning) pour créer des organisations « capacitantes » (Boboc et Metzger 2015).

$2474 \%$, selon le baromètre AIFNEF 2015, avec une satisfaction de 3,7 sur 5. Le MOOC reçoivent d'ailleurs l'indice de satisfaction le plus bas de cette enquête, qui est, rappelons-le, produite par les parties prenante de l'e-learning classique, éditeurs de textes et logiciels. 
En revanche, le «halo» des MOOC, qui illumine ses dérivés, permet l'établissement d'un nouveau jeu d'acteurs dans un domaine qui vit de cycles d'innovations "disruptives", comparable à "l'uberisation» dans d'autres secteurs comme le transport ou le logement. Les désormais «anciens» acteurs du monde des MOOC, comme Udacity, Coursera et edEx, se trouvent face aux nouveaux acteurs, comme, par exemple, les français Neodemia et Openclassrooms, d'anciens acteurs de l'e-learning qui élargissent leur portfolio. Ce qui est plus surprenant encore, c'est de voir émerger une nouvelle classe d'acteurs, notamment les récemment confirmés : les écoles, universités et leurs succursales, tentés par la création de spin-offs, transferts technologiques et de savoir-faire, à l'instar du modèle États-Unien ${ }^{25}$.

Certains de ces acteurs utilisent donc leur maîtrise des processus dans une sphère d'action rationnelle en finalité alors que d'autres, liés aux acteurs historiques de la formation à distance, renforcent la sphère d'action rationnelle en valeur, comme FUTURE LEARN (de l'Open University Britannique), qui développe particulièrement le versant networked learning de l'éducation ouverte en ligne, fidèle à leur engagement historique pour l'ouverture et l'égalité d'accès à l'éducation. Cet exemple, tout comme l'engagement du ministère de l'enseignement français dans le projet FUN, illustre l'implication d'institutions publiques dans un positionnement de l'éducation comme bien commun.

La plupart des acteurs professionnels de MOOC, entreprises de services organisant la distribution en co-production avec les universités et proposant des plateformes d'accès aux MOOC, ont dû préciser leur modèle économique. L'action rationnelle en valeur a été transformée en valeur tout court : Les MOOC premium offrent aujourd'hui, moyennant paiement, la dimension sociale si importante à travers l'accompagnement et des mentors et l'échange dans une communauté de pairs selon la stratégie " content is the bait, people the premium ${ }^{26}$. Ce développement contraste avec des orientations discursives des universitaires. Ainsi, selon Jansen et al. (2015), les établissements universitaires européens valorisent d'avantage la flexibilité de l'apprentissage et la possibilité d'inclure des publics jusque-là éloignés de l'université. Les établissements américains présents dans cette même étude mettent en avant des raisons financières et le recrutement d'étudiants. La visibilité de l'institution reste néanmoins la motivation principale de l'engagement dans les développements des MOOC sur les deux continents.

\section{Pour une approche communicationnelle des formations en ligne}

Nous avons insisté sur l'utilité de l'approche des médiacultures pour analyser des dispositifs techno-pédagogiques récents, tant leur mode de production

25 Udacity et Coursera émanent quant à eux initialement du contexte universitaire américain.

$26 \mathrm{https}: / /$ medium.com/thousandnetwork/lifelong-learning-5-inspirations-for-a-new-digital-workforce-ineurope-929aa48f187 
et de promotion partage ceux des industries culturelles ${ }^{27}$. Dès lors, il s'agit de comprendre l'objet comme un «site de tensions dynamiques » (Macé 2005) et de l'aborder avec les outils d'analyse des industries culturelles. Ceci suppose de prendre en compte les acteurs, l'environnement socio-économique et ses contraintes aussi bien que les dispositifs d'apprentissage en ligne et leurs régimes de visibilité dans une visée dispositive.

En tant que phénomène de la culture numérique le MOOC a permis l'amplification et la mise en circulation de débats autour de la culture d'apprentissage, l'avenir de l'éducation en tant que bien public et surtout de l'université en tant qu'institution. Avec notre retour initial sur le développement des technologies de la formation à distance nous avons voulu montrer la continuité d'une tension dans le domaine des technologies dites éducatives entre ce que nous pourrions nommer des éléments relevant respectivement de la sphère d'action rationnelle en valeur et de la sphère d'action rationnelle en finalité. Les technologies jouent ici le rôle de catalyseur et non d'origine des motivations d'action. Grâce à l'approche dispositive de l'univers des MOOC, incluant aussi bien l'outillage que les acteurs et leur contexte économique et social, nous avons pu constater que la collusion des deux modes normatifs et performatifs est récurrente dans ce secteur et explique en partie sa force d'attraction. Nous retrouvons ce phénomène dans beaucoup de domaines connexes, y compris des plus récents comme les Learning Analytics, analyse des traces des apprenants, qui servent tantôt à l'amélioration de la progression pédagogique des apprenants et tantôt au suivi marketing et comptable des institutions sans que lon puisse pour autant clairement distinguer les deux (Karsenti 2013).

Les divers dispositifs de formation informent quant aux relations entre groupes et individus, à tel ou tel moment de l'histoire des sociétés, ainsi que sur la construction des systèmes de représentation et la diffusion de référentiels communs. Ainsi nous proposons de penser la «transformation digitale» de la formation 2.0 à la fois comme un texte culturel et comme un dispositif problématisé appartenant à la fois à la sphère de l'action rationnelle en valeur et en finalité car c'est bien l'imbrication des deux qui est caractéristique et constitutive. C'est bien la rationalité communicationnelle des dispositifs en question et son caractère bi-face, qui permet ainsi de les situer et de comprendre leurs enjeux. Ces enjeux sont réactualisés mais s'insèrent dans une longue lignée de tension entre emplois contradictoires des technologies cognitives qui renvoie à une approche résolument dispositive intégrant des méthodes digitales et par leur ouverture aux apports des sciences de l'éducation et de la socio-histoire des médias.

27 Les travaux des SIC en ce domaine permettent de bénéficier d'un cadre conceptuel bien solide (Moeglin, Bouquillon, Legendre...). 


\section{BIBLIOGRAPHIE}

ACHERE C., " Histoire de la Fédération Interuniversitaire de l'Enseignement à Distance (FIED) », Distances et médiations des savoirs, Vol. 6, 2014, URL: http://dms. revues.org/719

AFINEF, Baromètre "l'e-learning en France ", 2015, URL : http://www.afinef.net/ barometre-e-learning

BAUDOIN E. et TAHSSAIN-GAY L., "Déploiement du e-learning dans le cadre de la formation en entreprise: quel rôle de l'environnement social dans l'engagement des salariés? " Recherches en Sciences de Gestion, Vol. 4, N 97, 2013, p.109-126.

BERTEN A., " Dispositif, médiation, créativité: petite généalogie », Hermès, 25, 1999, p.33-47.

BOBOC A., METZGER J.-L., «Penser la place du numérique dans la formation en entreprise : apports de la notion d' " environnement capacitant», Colloque scientifique international - «e-Formation des adultes et des jeunes adultes », Lille, 2015, Pré-actes URL : www.trigone.univ-lille1.fr/eformation2015/preactes/09.pdf

CHAPTAL A., L'efficacité des technologies éducatives dans l'enseignement scolaire: Analyse critique des approches française et américaine, Paris : L'Harmattan, 2003.

COLLET L. et WILHELM, C., dir., Numérique, éducation et apprentissage : Enjeux communicationnels, Paris: L'Harmattan, 2015.

DANIEL John S., Megauniversities and Knowledge-Media, London : Routledge, 1997.

DANIEL John S., "What Role for Open Universities when eLearning Becomes Universal?» in Future of Open and Distance Learning for Knowledge Network Society, Seoul: Korea National Open University, 2012, p. 9-71.

DANIEL John S., « MOOCs - Evolution or Revolution ? » Journal of Online Learning and Teaching, 10(1), 2014, p. 287-301.

DEPOVER C., "Quels modèles économiques et pédagogiques pour les MOOC ? ", Distances et médiations des savoirs, Vol. 5, 2014, URL: http://dms.revues.org/530

FLICHY P., L'imaginaire d'Internet, Paris : La Découverte, 2001.

GALIBERT O., «Vers une instrumentalisation généralisée du lien communautaire en ligne : la montée en puissance du community management » in Lépine, V., C., MilletFourrier et F. Martin-Juchat, Acteurs de la communication des entreprises et organisations : pratiques et perspectives. Grenoble, France : Presses universitaires de Grenoble, 2014, p. $117-136$.

HILGARD E. R. et BOWER G. H., Theories of learning. 3rd ed, New York: Appleton-Century-Crofts. Chapter 16: Learning \& the technology of instruction, 1966, p. 554-561.

HOLMBERG B., " Guided didactic conversation in distance education ».in D. Sewart, D. Keegan, et B. Holmberg (dirs.), Distance education: International perspectives, London: Croom Helm, 1983, p. 114-122.

HOLMBERG B., The Evolution of the Character and Practice of Distance Education. Open learning, 10(2), 1995, p. 47-53. 
JACQUINOT-DELAUNAY G. et CHOPLIN H., " La démarche dispositive aux risques de l'innovation ", Education Permanente, $\mathrm{N}^{\circ} 152$, 2002, p. 185-198.

JACQUINOT-DELAUNAY G., "Sic et Sed sont dans un bateau ... ". Hermès, n 38(1), 2004, p. 198-198.

JAILLET A., Lécole à l'ère numérique - Des Espaces Numériques pour l'éducation à l'Enseignement à Distance. Paris: L'Harmattan, 2004.

JAILLET A., "Les films promoteurs de MOOC, une rhétorique de la "divisio"», Distances et médiations des savoirs, 8, 2014, URL: http://dms.revues.org/951

JANSEN D. et al., «Comparing MOOC Adoption Strategies in Europe: Results from the HOME Project Survey", International Review of Research in Open and Distributed Learning, 16(6), 2015, p.116-136.

JEFFRIES M., Research in distance education, IPSE-Index of the Faculty Handbook, [en ligne], 2000,URL: http://www.computerschool.net/edu/DL_history_mJeffries.html

KARSENTIT., "MOOCs, révolution ou simple effet de mode? ", Revue internationale des technologies en pédagogie universitaire, 10(2), 2013, URL : www.ritpu.org

LARROCHE V., "Quelle visibilité professionnelle pour un salarié ambassadeur 2.0 auprès des recruteurs potentiels?», Communication et organisation, 44, 2013, p. 53-64.

MAIGRET E. \& MACE E., Penser les médiacultures. Nouvelles pratiques et nouvelles approches de la représentation du monde, Paris : Armand Colin-INA, 2005.

MIÈGE B., «L'espace public: perpétué, élargi et fragmenté», dans PAILLART Isabelle (sous la direction de), L'espace public et l'emprise de la communication, Grenoble : éditions Ellug, 1995, p. 163-175.

MIÈGE B., La société conquise par la communication. Tome 2: La communication entre l'industrie et l'espace public, Grenoble: éditions des Presses Universitaires de Grenoble, collection Communication, médias et sociétés, 1997.

MOEGLIN P., Les industries éducatives, Paris, PUF, 2010.

MOEGLIN P., «L'enseignement supérieur au défi du numérique - MOOC: de l'importance d'un épiphénomène». Futuribles, n³98, 2014, URL: https://www. futuribles.com/fr/revue/398/lenseignement-superieur-au-defi-du-numerique-mooc-/

NOBLE D. F., Digital diploma mills : the automation of higher education. New York: Monthly Review Press, 2001.

OBLINGER D., dir., Game Changers: Education and Information Technologies, Educause, 2012, URL : http://net.educause.edu/ir/library/pdf/pub7203.pdf

PERRIAULT J., L'accès au savoir en ligne. Paris: Odile Jacob, 2003.

PRESSEY S. L., Psychology and the new education. Harper, 1933.

SAETTLER, P. A., History of Instructional Technology. New York: McGraw-Hill, 1968.

THIBAULT F., «Divorcer du technicisme. Une histoire de l'enseignement supérieur à distance en France », Distances et savoirs 3/5, 2007, p. 367-391.

WALLET J., «À l'heure de la société mondialisée du savoir, peut-on supprimer les enseignants ? "Hermès, 45, 2006, p. 91-98.

WEBER M., Wirtschaft und Gesellschaft (1. Halbband). Köln, Berlin: Kiepenheuer \& Witsch, 1922/1964. 
WILHELM C., «MOOC et SIC - une relation propice à une synthèse méthodologique, dans Education et Apprentissage et SIC », in Numérique, éducation et apprentissage (dir. Laurent Collet et Carsten Wilhelm): Enjeux communicationnels. Paris: L'Harmattan, 2015.

WILHELMC., "Approche dispositive et triangulation : mots-clés d'une méthodologie SIC interdisciplinaire en contexte interculturel », XVII Congrès de la Société des Sciences de l'Information et de la Communication : "Au cœur et aux lisières des SIC» Dijon, 23-25 juin 2010, p. 279-286, URL : http://fr.calameo.com/books/0007559198827faf88a13

WILHELM C., "Émergence d'une culture communicationnelle au sein d'un dispositif international en ligne. Distances géoculturelles et proximité axiologique», Distances et savoirs, Vol. 8/1, 2010, p. 79-107, URL : www.cairn.info/revue-distances-etsavoirs-2010-1-page-79.htm

Résumé : La constance de la promesse de rupture autour des phénomènes de formation en ligne récents tels que les MOOC cristallise une tension dynamique inhérente au domaine. Pour soutenir notre argument que les dispositifs dérivés dans la formation en entreprise jouissent d'un « effet halo » ou auréole établi par le discours d'accompagnement des MOOC, nous revenons sur la place des technologies dans la formation à distance afin de montrer que la tension entre action rationnelle en finalité et en valeur est caractéristique pour le secteur de la formation. L'approche communicationnelle est le prisme qui nous permet de comprendre ce phénomène médiaculturel dont le fonctionnement est proche des productions des industries culturelles.

Mots-clés: rationalité communicationnelle, MOOC, médiacultures, formation à distance, effet halo.

Abstract: The insistence on the promise of disruption in the promotion of recent phenomena in distance learning such as MOOCs crystallizes a dynamic tension inherent to the field. To support our argument that the forms of digital learning in today's private sector profit largely from the "halo effect" of MOOCs we propose to go back to the imbrication of technological advances in distance learning to show that the tension between a value-oriented rationality and a goal-oriented rationality is characteristic of the field. We propose to look at these mediacultural phenomena, whose functioning resembles the culture industries, through the prism of communicative rationality.

Keywords : communicative rationality, MOOC, mediacultures, distance learning, halo effect. 